\title{
Block hybrid-like method for solving delay differential equations
}

\begin{abstract}
The block hybrid-like method is presented and implemented in predictor-corrector mode to solve delay differential equations. The Lagrange interpolation polynomial is implemented to obtain the approximation for the delay terms. The Q-stability of the method is investigated. The illustrative examples are presented to demonstrate the efficiency of the block hybrid-like method.
\end{abstract}

Keyword: Block; Delay differential equations; Hybrid 